\title{
FORMATION OF GIANT PLANETS - AN ATTEMPT IN MATCHING OBSERVATIONAL CONSTRAINTS
}

\author{
YANN ALIBERT, CHRISTOPH MORDASINI, OLIVIER MOUSIS and WILLY BENZ \\ Physikalisches Institut, University of Bern, CH-3012 Bern, Switzerland
}

Received: 1 June 2004; Accepted in final form: 13 October 2004

\begin{abstract}
We present models of giant planet formation, taking into account migration and disk viscous evolution. We show that migration can significantly reduce the formation timescale bringing it in good agreement with typical observed disk lifetimes. We then present a model that produces a planet whose current location, core mass and total mass are comparable with the one of Jupiter. For this model, we calculate the enrichments in volatiles and compare them with the one measured by the Galileo probe. We show that our models can reproduce both the measured atmosphere enrichments and the constraints derived by Guillot et al. (2004), if we assume the accretion of planetesimals with ices/rocks ratio equal to 4 , and that a substantial amount of $\mathrm{CO}_{2}$ was present in vapor phase in the solar nebula, in agreement with ISM measurements.
\end{abstract}

Keywords: Stars: planetary systems - stars: planetary systems: formation - solar system: formation

\section{Introduction}

The current paradigm for the formation of giant gaseous planets is based on the so-called core accretion model in which a growing solid core reaches a critical mass and accretes rapidly a massive atmosphere (Pollack et al., 1996). This model explains for example the global enrichment in heavy elements observed in the giant planets, and can be used to interpret the enrichments in volatiles observed in the atmosphere of Jupiter by the mass spectrometer on-board the Galileo probe (Atreya et al., 1999; Mahaffy et al., 2000). However, while this model has many appealing features, it suffers at least from three shortcomings.

First, the timescale (close to $10 \mathrm{Myr}$ ) found by Pollack et al. (1996) to form Jupiter at its present location is uncomfortably close to the lifetime of protoplanetary disks which is believed to be of the order of 1-10 Myr (Haisch et al., 2001). This timescale problem has led others to look for more rapid formation mechanisms based on direct gravitational collapse (Boss, 2002; 2004). Second, Pollack et al. (1996) assumed that the giant planets of our solar system have been formed where they are observed today. However, the discovery over the last decade of extrasolar planets at very short distances to their host star has opened the possibility that planets may actually migrate over large distances (Lin et al., 1996; Trilling et al., 1998; Papaloizou and Terquem, 1999). The time scale of migration is still very uncertain, but conservative estimates give values between 0.1 and $10 \mathrm{Myr}$. 
Finally, the interpretation by Gautier et al. (2001a; b) and Hersant et al. (2004) of the enrichment in volatiles in Jupiter is not strictly speaking consistent with Pollack et al. (1996). These authors assume, for example, that all planetesimals have been accreted by Jupiter during the late hydrodynamical phase (phase 3 in Pollack et al., 1996), although nearly one half are accreted during phase 1 and phase 2.

Since all relevant timescales (planet formation, disk evolution, and migration) are of the same order of magnitude, it appears difficult to obtain a self-consistent model while omitting anyone of these processes. In Section 2 below, we briefly summarize our efforts to develop such a self-consistent model within the framework of the core-accretion scenario. New formation models of giant planets including these processes are presented in Section 3. In Section 4, we will use these models to calculate the corresponding volatile enrichment and will compare them to the observed one in Jupiter. A summary and conclusions are presented in Section 5.

\section{The Model}

Basically, our model to compute the formation of giant planets follows closely the work by Pollack et al. (1996) with some notable addition. It consists in three different modules that calculate: 1) the disk structure and its time evolution, 2) the interaction of planetesimals with the atmosphere of the planet, and 3) the internal structure of the planet. We give here a short description of each module. More details and some tests of the model can be found in Alibert et al. (2004a).

\subsection{Disk Structure ANd Evolution}

Contrary to Pollack et al. (1996), we do not assume a static disk but rather a time evolving one. For simplicity, we assume a so-called $\alpha$-disk (axisymmetric and with constant $\alpha$ ) for which we determine the structure (both vertical and radial) as a function of time using the method described in Papaloizou and Terquem (1999). From the vertical structure, we compute, as a function of distance to the star $r$, the surface density $\Sigma$ and subsequently the mid-plane temperature and pressure $T_{\text {mid }}(r, \Sigma), P_{\text {mid }}(r, \Sigma)$ the mean viscosity $v(r, \Sigma)$, and the disk density scale height $H(r, \Sigma)$. The first two quantities are needed as boundary conditions in the calculation of the internal structure of the planet, whereas the two others enter in the calculation of the radial structure of the disk. The time evolution of the disk is governed by a diffusion equation, modified to take into account the momentum transfer between the planet and the disk, as well as the effect of photo-evaporation. The rate of momentum transfer between the planet and the disk is calculated following Lin and Papaloizou (1986)

$$
\Lambda(r)=\frac{f_{\Lambda}}{2 r} \sqrt{G M_{\text {star }}}\left(\frac{M_{\text {planet }}}{M_{\text {star }}}\right)^{2}\left(\frac{r}{\max (|r-a|, H)}\right)^{4},
$$


where $a$ is the sun-planet distance and $f_{\Lambda}$ is a numerical constant. The photoevaporation term is as in Veras and Armitage (2003).

\subsection{Migration}

Gravitational interactions between the growing protoplanet and the disk lead to inward migration and possibly gap formation (Lin and Papaloizou, 1986; Ward, 1997; Tanaka et al., 2002). For low mass planets, the migration rate is linear with mass (type I migration, Ward, 1997). Higher mass planets open a gap and the migration rate is set by the viscosity (type II migration, Ward, 1997).

While the general physical understanding of the origin of migration is clear, the actual migration rates obtained for type I migration especially are so short that all planets should actually be destroyed by the central star long before the disappearance of the gaseous disk. Tanaka et al. (2002) have performed new analytical calculations of type I migration, in two or three dimensional disks and found longer migration timescales but unfortunately still too short to ensure survival. Further suggestions for increased type I migration timescales can be found in calculations by Nelson and Papaloizou (2003). As suggested by these authors, torques exerted on at least small mass planets $\left(M_{\text {planet }}<30 M_{\oplus}\right)$ embedded in turbulent MHD disks are strongly fluctuating resulting in a slow down of the net inward motion. Moreover, as shown by Menou and Goodman (2003), type I migration of low-mass planets can be slowed down by nearly one order of magnitude in regions of opacity transitions.

These considerations seem to indicate that the actual migration timescales may in fact be considerably longer than originally estimated by Ward (1997) or even by Tanaka et al. (2002). For these reasons, and for lack of better knowledge, we actually use for type I migration the formula derived by Tanaka et al. (2002) reduced by an arbitrary numerical factor $f_{I}$ (set to $1 / 30$ in this paper). Tests have shown that provided this factor is small enough to allow planet survival, its actual value does not change the formation timescale but just the extend of migration (see Section 3.3).

For type II migration, two cases have to be considered. For low mass planets (when their mass is negligible compared to the one of the disk) the inward velocity is given by the viscosity of the disk. When the mass of the planet becomes comparable to the one of the disk, migration slows down and eventually stops. In this latter case, the variation of the planet's orbital momentum is equal to the angular momentum transport rate (Lin and Papaloizou, 1985; Lin et al., 1996):

$$
\frac{d}{d t}\left[M_{\text {planet }} a_{\text {planet }}^{2} \Omega\right]=\frac{3}{2} \Sigma \nu \Omega r^{2},
$$

where $\Omega^{2}=G M_{\text {star }} / a_{\text {planet }}^{3}$, and the second term is calculated at the current position of the planet, $a_{\text {planet }}$, but using the non perturbed gas surface density. In all cases of type II migration, the migration rate is limited to the viscous velocity of the disk. 
Migration type switches from type I to type II when the planet becomes massive enough to open a gap in the disk which occurs when the Hills radius of the planet becomes greater than the density scale height $\mathrm{H}$ of the disk.

\subsection{The Planetesimals}

In this module we compute the trajectory, the energy and mass loss of planetesimals falling through the atmosphere of the planet under the influence of gravity and aerodynamic drag forces. The drag coefficient is calculated (assuming a sphere) as a function of the local Mach and Reynolds numbers using the equations given by Henderson (1976). The loss in kinetic energy results in a local heating of the planet's atmosphere which enters in the calculation of the internal structure. Given the size of the planetesimals considered here $(100 \mathrm{~km})$, ablation is found to be negligible and deposition of mass occurs almost entirely due to fragmentation which occurs when the pressure at the stagnation point becomes larger than the planetesimals tensile strength. We do not take into account any diffusion effects that could bring planetesimals from outside to inside the feeding zone.

Due to the scattering effect of the planet, the surface density of planetesimals is constant within the current feeding zone but decreases with time proportionally to the mass accreted (and/or ejected from the disk) by the planet.

The feeding zone is assumed to extend to a distance of $4 R_{\text {Hill }}$ on each side of the planetary orbit, where $R_{\text {Hill }}$ is the Hills radius of the planet. We use the expressions in the Appendix B and C of Greenzweig and Lissauer (1992) to calculate the gravitational enhancement factor.

For the inclination and eccentricities of the planetesimals we adopt the same values as in Pollack et al. (1996). For the physical properties of the planetesimals we use the values for ice.

Finally, it is important to take into account the ejection of planetesimals scattered by the planet since the amount of planetesimals ejected from the feeding zone directly determines the final abundance of heavy elements in the planet. To compute the ejection rate, we use the accretion to ejection ratio scaling derived from Ida and Lin (2004):

$$
f_{\text {acc }}\left(\frac{V_{\text {Kepl }}}{V_{\text {esc, planet }}}\right)^{4},
$$

where $V_{\text {Kepl }}$ is the disk keplerian velocity at the planet location, and $V_{\text {esc,planet }}$ the escape velocity from the planet. This latter formula is an order of magnitude estimate for the ejection rate, and is still subject to some uncertainties. However, work by Guillot and Gladman (2000) suggests that the ejection rate may be high enough to prevent the accretion of more than $\sim 8 M_{\oplus}$ on a $10 M_{\oplus}$ core, at the present location of Jupiter. This suggests that the ejection rate may be signicantly higher than the one given by Equation (3) using $f_{\text {acc }}=1$. 
Ongoing $N$-body calculations (Horner et al., 2004) suggest a value of $f_{\text {acc }}$ of the order of 10 to 100 depending on the planet's location and planet's mass. We will assume in the results presented here $f_{\text {acc }}=10$.

\subsection{Protoplanet StruCture AND Evolution}

The internal structure of the planet is calculated, taking into account a growing core (and the inner luminosity due to the accretion of planetesimals). We use for the models presented here the sinking approximation described in Pollack et al. (1996): after dissolution inside the envelope, planetesimals debris are assumed to slowly sink to the core, leading to an extra term in the luminosity. This corresponds to the approximation used in the standard case of Pollack et al. (1996). As shown by Alibert et al. (2004a), planetesimals are destroyed relatively deep in the envelope. Consequently, the differences between the sinking and the no sinking approximation are found to be much smaller than in Pollack et al. (1996).

The equations of planet evolution are solved, using opacities from Bell and Lin (1994) and the equation of state from Chabrier et al. (1992). The outer boundary conditions are given by requiring that the disk and the planet join smoothly at the outer radius, i.e. $T_{\text {surf }}=T_{\text {mid }}(r, \Sigma)$, and $P_{\text {surf }}=P_{\text {mid }}(r, \Sigma)$. The gas accretion rate onto the planet is determined by the condition: $R_{\text {planet }}=\min \left(R_{\text {Hill }}, R_{\text {acc }}\right)$ where $R_{\text {planet }}$ is the outer radius of the planet, and $R_{\text {acc }}$ the accretion radius (see Pollack et al., 1996). At each timestep, we calculate the mass of the envelope required to reach this condition. However, in reality, the latter condition can only be satisfied if the disk can actually supply enough gas to the planet. Once a gap in the disk opens, the maximum gas accretion rate is set to the rate given by Veras and Armitage (2003). At this stage, the growth in mass of the planet is set by the disk and no longer by the internal structure of the planet which is no longer computed.

\section{New Formation Models}

\subsection{IN SITU FORMATION}

To properly quantity the effect of migration on planet formation, we first compute a model in which migration is turned off. Figure 1 shows the mass of accreted planetesimals as well as the mass of gas as a function of time for a planet at 5.5 $\mathrm{AU}$. The gas and solid surface densities are non evolving in this simulation, and their values are $525 \mathrm{~g} / \mathrm{cm}^{2}$ and $10 \mathrm{~g} / \mathrm{cm}^{2}$. As in Pollack et al. (1996), we do not consider the ejection in this simulation and the next one.

This model corresponds approximately to case J2 of Pollack et al. (1996), the corresponding timescale for formation being around $50 \mathrm{Myr}$ in their simulation. The three phases described in Pollack et al. (1996) are clearly identified, the timescale for formation is given by the length of phase 2 . The formation time is around 30 Myr, somewhat lower than in Pollack et al. (1996), but still much longer 


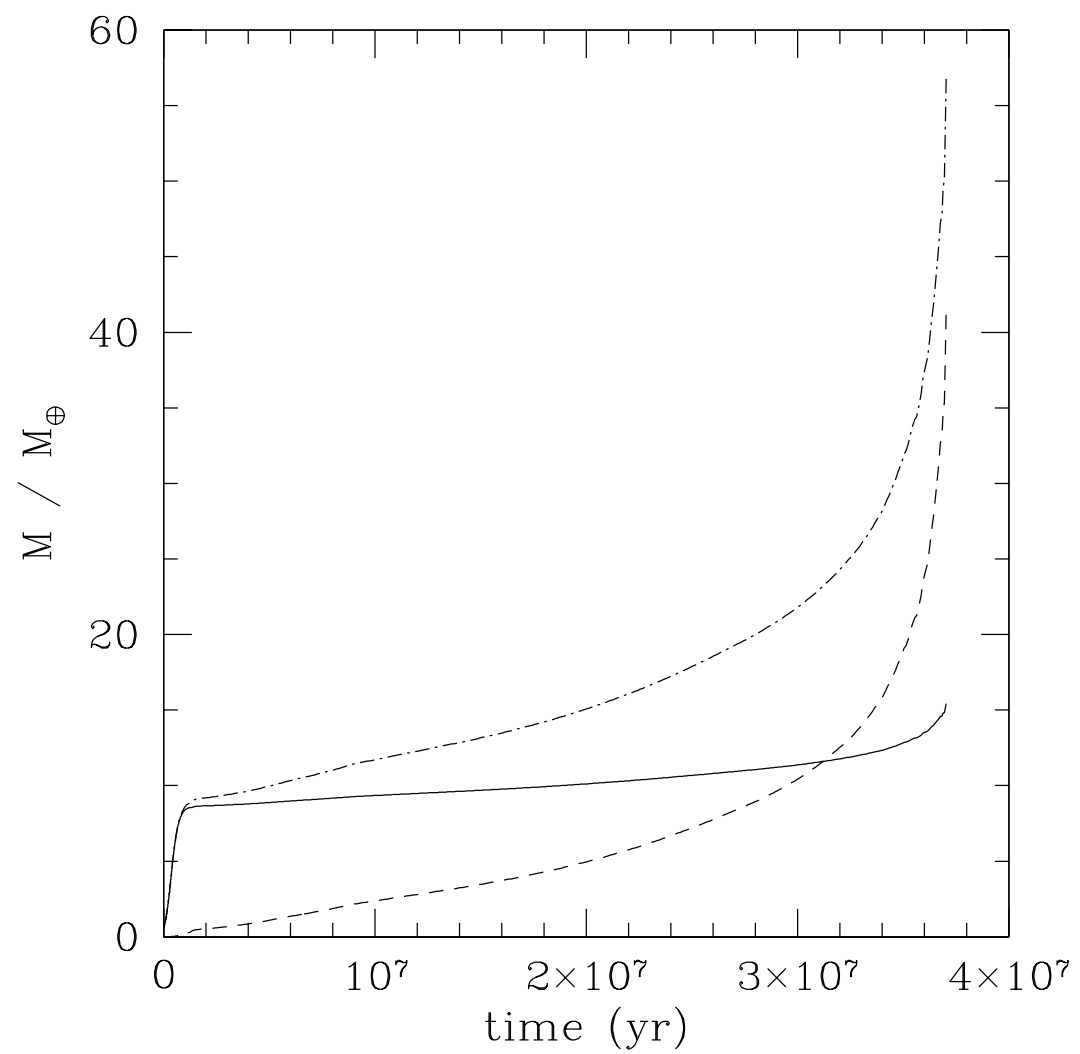

Figure 1. Time evolution of the planet in the case of in-situ formation. Solid line: Mass of accreted planetesimals (either in core or dissolved in the envelope), dashed line: mass of accreted gas and dot-dashed line: total mass.

than typical disks lifetimes. The difference with Pollack et al. (1996) provides a measure for the sensitivity of the results to differences in physical and numerical approximations used in both approaches. In particular, we use a different equation of state and opacity law (which has been shown to have a huge influence on the length of phase 2, see Pollack et al., 1996), and our initial model is not exactly the one of Pollack et al. (1996).

\subsection{INITIAL Disk MODELS}

To calculate models with migration and disk evolution, we have to specify an initial disk profile, as well as the disk viscosity. We will consider two kinds of initial surface density profiles, $\Sigma \propto r^{-2}$, and $\Sigma \propto r^{-3 / 2}$, where $r$ is the distance to the sun. The viscosity parameter $\alpha$ is set to $2 \times 10^{-3}$ which yields a typical evolution time of the disk of a few Myr. The gas-to-dust ratio is equal to 70 for disk mid-plane temperature below $170 \mathrm{~K}$, and 280 in the opposite case. The numerical parameters are $f_{I}$ (reduction of type I migration) equal to $1 / 30$ and $f_{\Lambda}$, the numerical factor 
in the expression of the momentum transfer between the planet and the disk, set to 0.05 . This latter choice gives a reduction of $\Sigma$ due to momentum transfer around $30 \%$ when the disk density scale height equals the Roche radius of the planet (corresponding to the moment when migration switches from type I to type II). The main conclusions presented here remain valid if $f_{\Lambda}$ is set to 0 : the effect of gap formation on formation is essentially due to the limitation of the accretion rate of gas (see Section 2.4), and not due to the variation of the boundary conditions at the surface of the planet.

\subsection{Formation with Migration: Timescale to Accrete a Massive ENVELOPE}

We first calculate the timescale to reach the crossover mass (mass of accreted planetesimals equal to mass of accreted gas). We consider an initial disk density profile given by $\Sigma \propto r^{-2}$, the constant being chosen to yield $\Sigma=525 \mathrm{~g} / \mathrm{cm}^{2}$ at $5.2 \mathrm{AU}$. As in Pollack et al. (1996), this surface density profile is chosen to have isolation masses that do not depend on the distance to the sun. In this section, we do not take into account photo-evaporation nor ejection, and we start with an embryo of $0.6 M_{\oplus}$ initially at $8 \mathrm{AU}$. Figure 2 shows the mass of planetesimals and the mass of gas accreted by the planet as a function of time. Note that the mass of accreted planetesimals does not correspond to the core mass since some fraction of them are being destroyed while traversing the envelope and never reach the core.

As in Pollack et al. (1996), the formation timescale is essentially determined by the time necessary to reach the runaway accretion phase which occurs shortly after the crossover mass (mass of core equals mass of envelope), $M_{\text {cross }}$, has been reached. Allowing for migration and disk evolution, we obtain a formation time of about $\sim 1$ Myr, i.e. thirty times faster than in our identical model in which migration and disk evolution have been switched off. The main reason for this speed-up is that owing to migration, the feeding zone is not as severely depleted as in Pollack et al. (1996), and hence, the long time needed to reach critical core mass and start runaway gas accretion is suppressed. Taking into account migration, the moving planet always encounters new planetesimals and thus its feeding zone is never emptied. To illustrate this important point, Figure 3 shows the initial and final disk profiles (for both the gaseous and the solid component).

Comparing a planet formed in situ or allowed to migrate at times of equal core mass (beginning of the simulation and after $0.7 \mathrm{Myr}$ ), we note that the envelope mass is always larger in the migrating case. This effect can also contribute to the speed-up, and can be understood as the combination of two effects (see Papaloizou and Terquem, 1999):

1. for a given core mass and distance to the star, the envelope mass increases when the accretion rate decreases

2. at a fixed accretion rate and core mass, the mass of the envelope is a growing function of the distance to the star. 


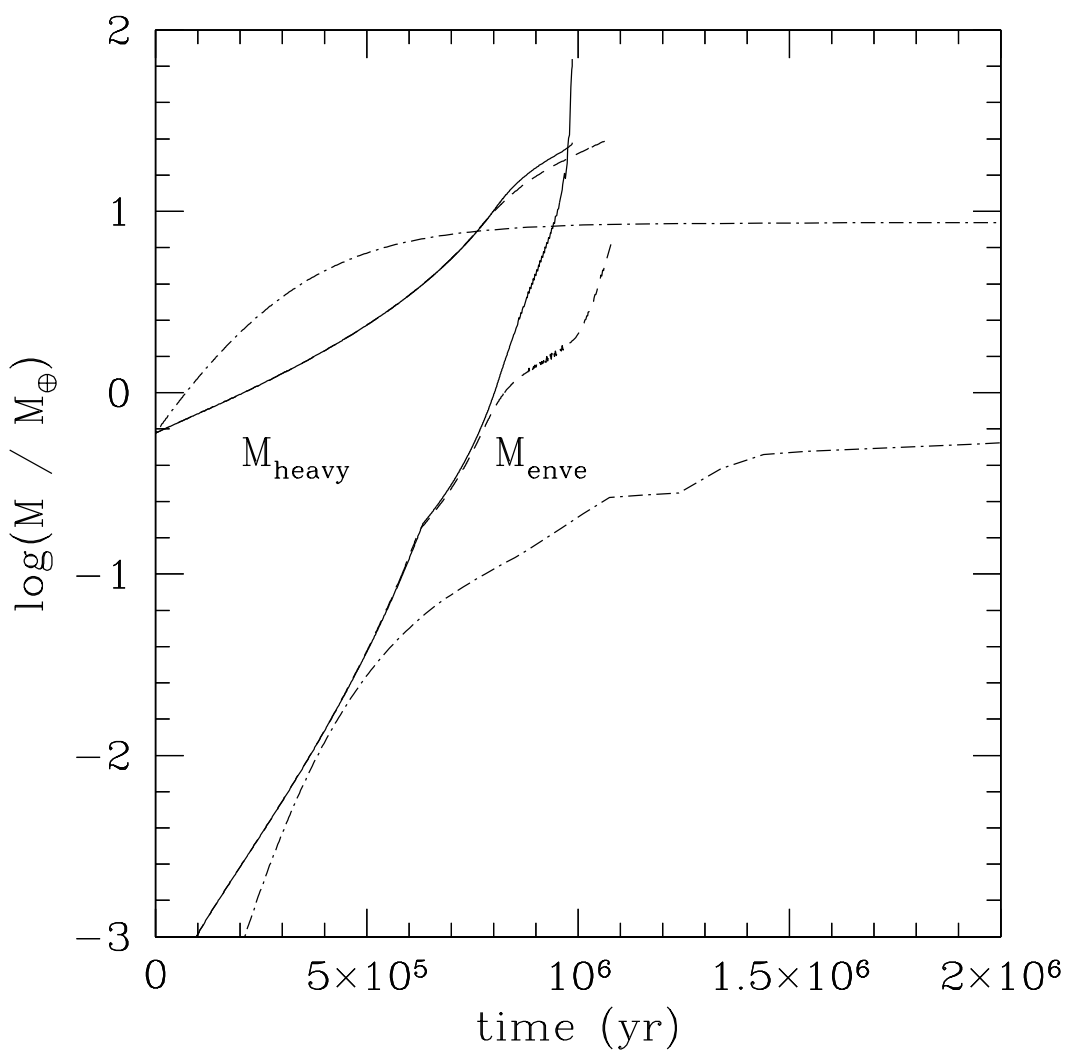

Figure 2. Mass of accreted planetesimals and mass of gas as a function of time for different models (all with initial disk surface density $\Sigma \propto r^{-2}$ ). Solid line: with migration but without gap formation, dashed line: with migration and gap formation, dot-dashed line: without migration nor gap formation (same as Figure 1).

This speed-up effect appears to be very robust against changes in the value of $f_{I}$ (reduction of type I migration), provided its value is small enough to ensure survival of the planet. For example, in a calculation in which the reduction of type I migration $\left(f_{I}\right)$ is set to 0.1 , an embryo starting at $15 \mathrm{AU}$ undergoes runaway accretion in less than $3 \mathrm{Myr}$. Reducing type I migration by 0.01 leads an embryo initially at $7 \mathrm{AU}$ to runaway after less than $1 \mathrm{Myr}$.

\subsection{Formation of a Giant Planet at 5 AU}

Depending on the different physical parameters used, a wide variety of giant planets can be formed. As an example, we provide here the result of a simulation yielding a giant planet of mass $\sim 350 M_{\oplus}$ located at about $5 \mathrm{AU}$ within less than 3 Myr. In this example, we consider a density profile, $\Sigma \propto r^{-3 / 2}$ normalized so that the mass of the disk (between $0.5 \mathrm{AU}$ and $50 \mathrm{AU}$ ) is $0.04 M_{\odot}$, and a disk photo-evaporation rate $10^{-8} M_{\odot} / \mathrm{yr}$. The type I reduction parameter is taken equal 


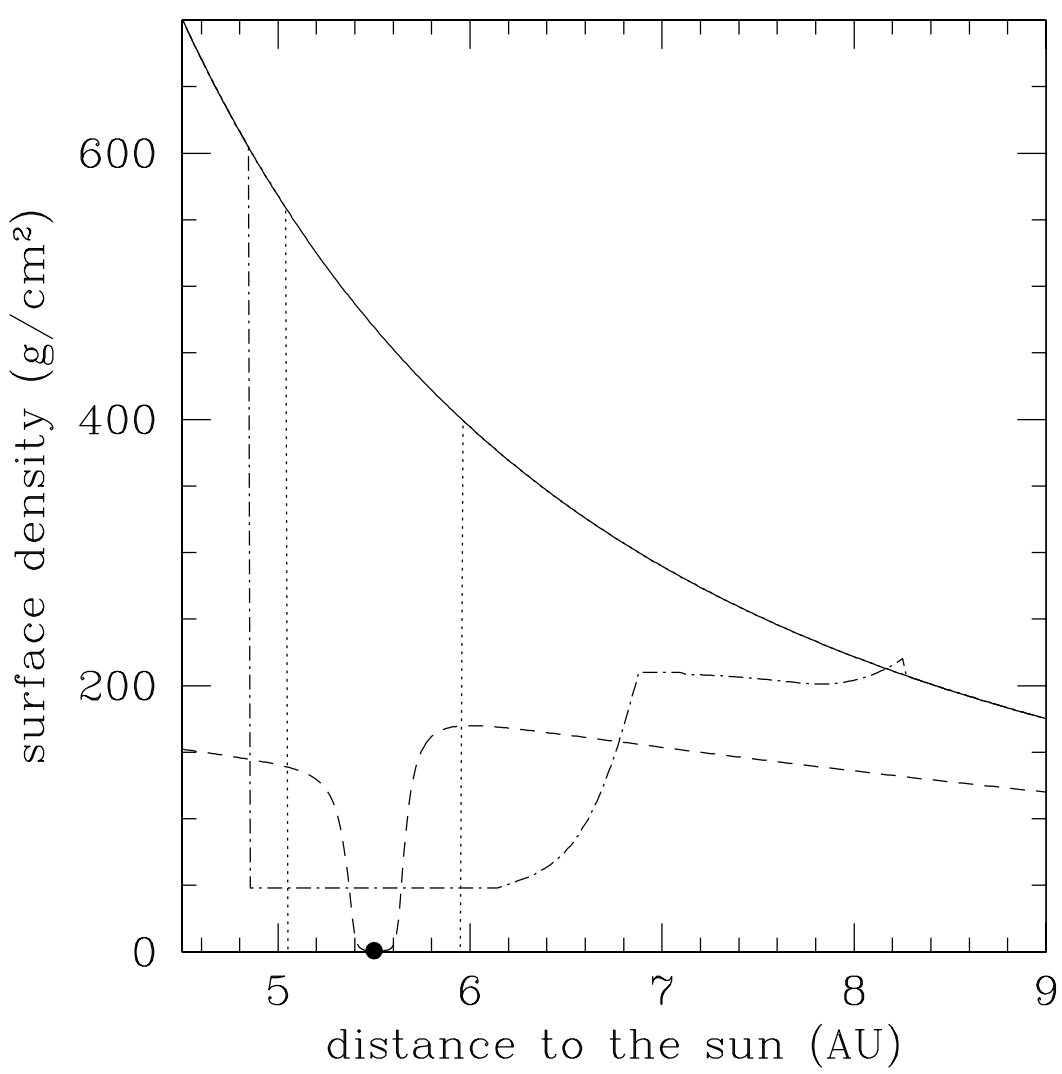

Figure 3. Gas and solid surface densities for the models with and without migration (both with an initial surface density $\Sigma \propto r^{-2}$ ). Solid line: initial surface densities; dashed line: gas surface density for model with migration and gap formation, after $1 \mathrm{Myr}$; dot-dashed line: solid surface density for the same model, at the same time; dotted line: solid surface density at the same time, but for the in-situ model. The solid surface densities are multiplied by 70 , and the big dot gives the position of the planet.

to $1 / 100$, and we do not take into account the effect of gap formation on the boundary conditions at the surface of the planet*. We start the calculation with an embryo at 11.5 AU. Figure 4 shows the evolution of the mass of the gaseous envelope, the mass of accreted planetesimals, as well as the mass of the disk, and Figure 5 gives the distance to the sun as a function of time. The crossover mass is reached after 1.6 $\mathrm{Myr}$, and at the same time, due to gap formation, the accretion rate of gas is limited to its maximum value, which decreases with decreasing disk mass. The formation process ends after less than $3 \mathrm{Myr}$ when the disk has disappeared. The final planet is characterized by a core of $\sim 6 M_{\oplus}$ and an envelope of $\sim 360 M_{\oplus}$ of which $\sim 36 M_{\oplus}$ are accreted planetesimals which were destroyed before reaching the core. This final mass of heavy elements may be slightly increased further during the

* however, the gap has still the effect of limitation of the gas accretion rate 


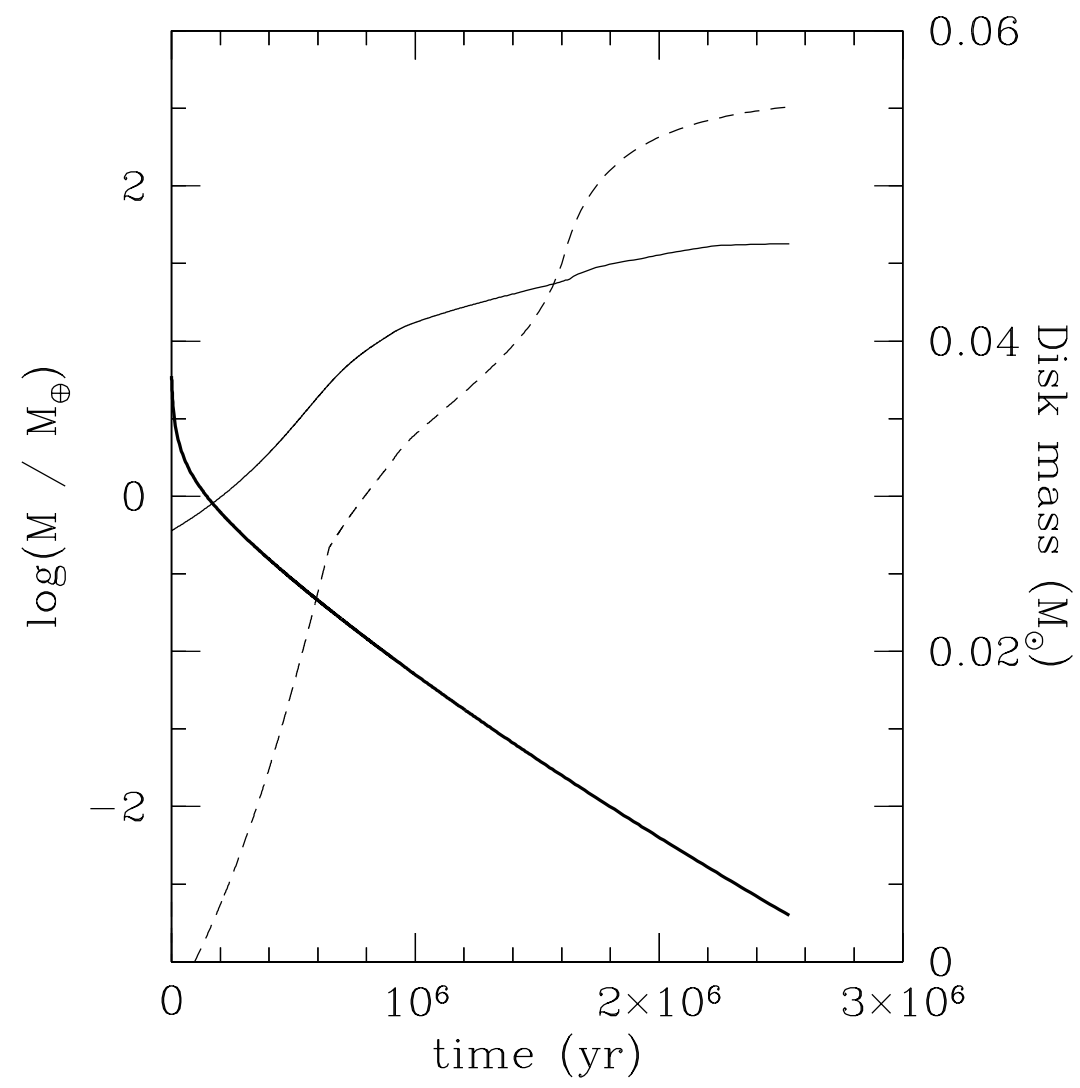

Figure 4. Mass of the different components for model of Section 3.4. Solid line: mass of accreted planetesimals, dashed line: mass of accreted gas, and heavy solid line: mass of the disk.

late stages of the formation, when the planet will accrete and/or eject the remaining planetesimals inside its final feeding zone. However, given the uncertainty on the ejection rate, the final content of heavy elements is not strongly constrained.

The migration of the planet can be divided into three phases. Before $\sim 1 \mathrm{Myr}$ the planet undergoes type I migration at which time migration switches to type II. Shortly before the end of the formation process, at $\sim 2.5 \mathrm{Myr}$, the mass of the planet becomes non negligible compared the disk mass and migration slows down and eventually stops when the disk has disappeared. At the end of the simulation, the planet is located at $5.14 \mathrm{AU}$, but it is expected that it will move to $\sim 5 \mathrm{AU}$ before the disk has completely disappeared.

Note that the extent of migration, and therefore the starting location of the embryo for a fixed endpoint, strongly depends upon the type I migration rate $\left(f_{I}\right)$. A higher rate $\left(f_{I}=0.03\right)$ results in the formation of a similar planet from an embryo initially located at $\sim 15 \mathrm{AU}$. Reducing the type I migration rate would also reduce the starting location of the embryo. 


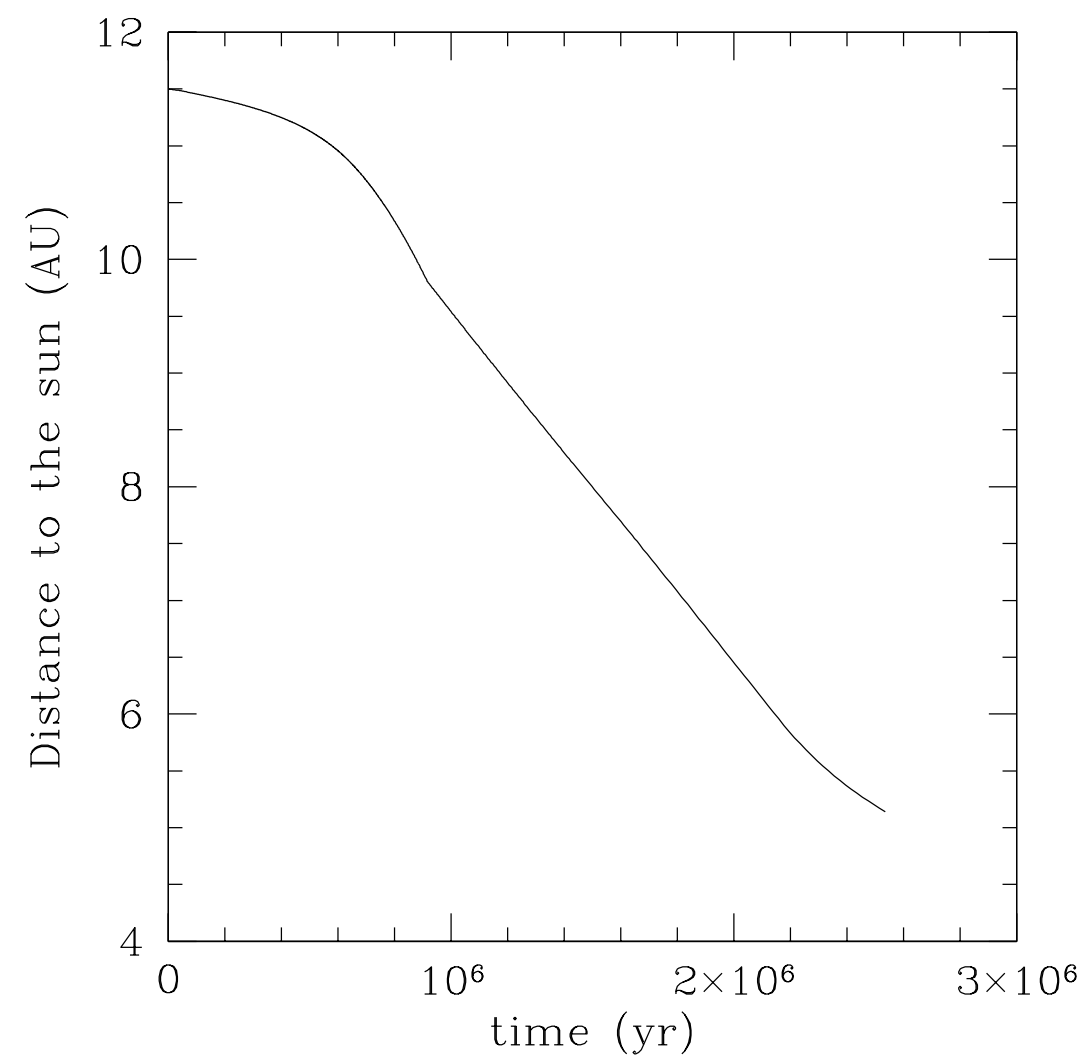

Figure 5. Distance to the sun for model of Section 3.4. The kink around $1 \mathrm{Myr}$ signals the change from type I to type II migration.

\section{Comparison with Observational Constraints from Jupiter}

We now consider the model of Section 3.4 which has a core and a total mass comparable to Jupiter and is located at a similar distance to the sun.

\subsection{Core Mass vs. Total Mass of Heavy Elements}

The total mass of heavy elements present inside the atmosphere of the planet is $\sim 36 M_{\oplus}$, coming from the accretion of planetesimals that have been destroyed inside the envelope before reaching the core*. The total mass of heavy elements inside the atmosphere is then compatible with the one derived by Guillot $e t$ al. (2004), whereas the mass of the core seems slightly too high. We obtain a core mass of $\sim 6 M_{\oplus}$, whereas the maximum core mass compatible with Guillot et al.

* One should also take into account some heavy elements accreted with the gas, but their abundance in the gas after planetesimals formation is highly reduced and therefore this contribution remains small. 
(2004), for our total mass of heavy elements, is of the order of $\sim 5 M_{\oplus}$. However, this mass depends on the assumed physical properties of the planetesimals. For example, considering some reduced tensile strength (for example due to voids or failures in planetesimals) would reduce this value. Moreover, the mass of the core may be reduced further if it dissolves to some extend during the evolution of the planet (Guillot et al., 2004).

Finally, we note again that the total mass of heavy elements depends on the ratio of planetesimal ejection versus accretion rate which can become quite large at late time.

\subsection{Volatile ENRICHMENTS IN THE ATMOSPhERE OF JUPiteR}

In order to interpret the volatile enrichments measured in Jupiter by Atreya et al. (1999) and Mahaffy et al. (2000), Owen et al. (1999) proposed that the heavy elements in Jupiter were acquired from the delivery of planetesimals formed from amorphous ices. Hence, they suggested that either Jupiter was formed beyond 30 AU before migrating to its actual position, or that the solar nebula was substantially cooler at $5 \mathrm{AU}$ than described by popular models, or that the heavy elements were delivered by planetesimals originating from distant regions. On the other hand, Gautier et al. (2001a; b) and Hersant et al. (2004) taking into account the in situ scenario of Pollack et al. (1996), proposed that volatiles were trapped as clathrate hydrates or hydrates in planetesimals located in the feeding zone of the giant planet. In order to model the corresponding enrichments of Jupiter's envelope, they assumed that most of solids were collected by the giant planet during the hydrodynamical collapse phase, at the end of the formation process, which is not strictly speaking consistent with Pollack et al. (1996).

In this subsection, we calculate the theoretical enrichments of $\mathrm{Ar}, \mathrm{Kr}, \mathrm{Xe}, \mathrm{C}, \mathrm{N}$, and $\mathrm{S}$ in our Jupiter-like planet computed in section 3.4 and compare these values to those observed for Jupiter. For this, we assume that volatiles rich planetesimals were formed from ices crystalized in the solar nebula. These planetesimals are continuously accreted during the planet migration, as calculated by our code.

Figure 6 describes the mass evolution of proto-Jupiter as a function of the distance to the Sun, for the model of Section 3.4. It can be seen that planetesimals start to accumulate onto proto-Jupiter as soon as migration begins. Accretion lasts until the planet reaches $5 \mathrm{AU}$. Volatiles are assumed to have been trapped in these planetesimals under the forms of hydrates, clathrate hydrates or pure ices during the initial cooling phase of the solar nebula, which is not modeled yet in our disk calculations. This phase is assumed to occur before the formation of the $0.6 M_{\oplus}$ embryo. 


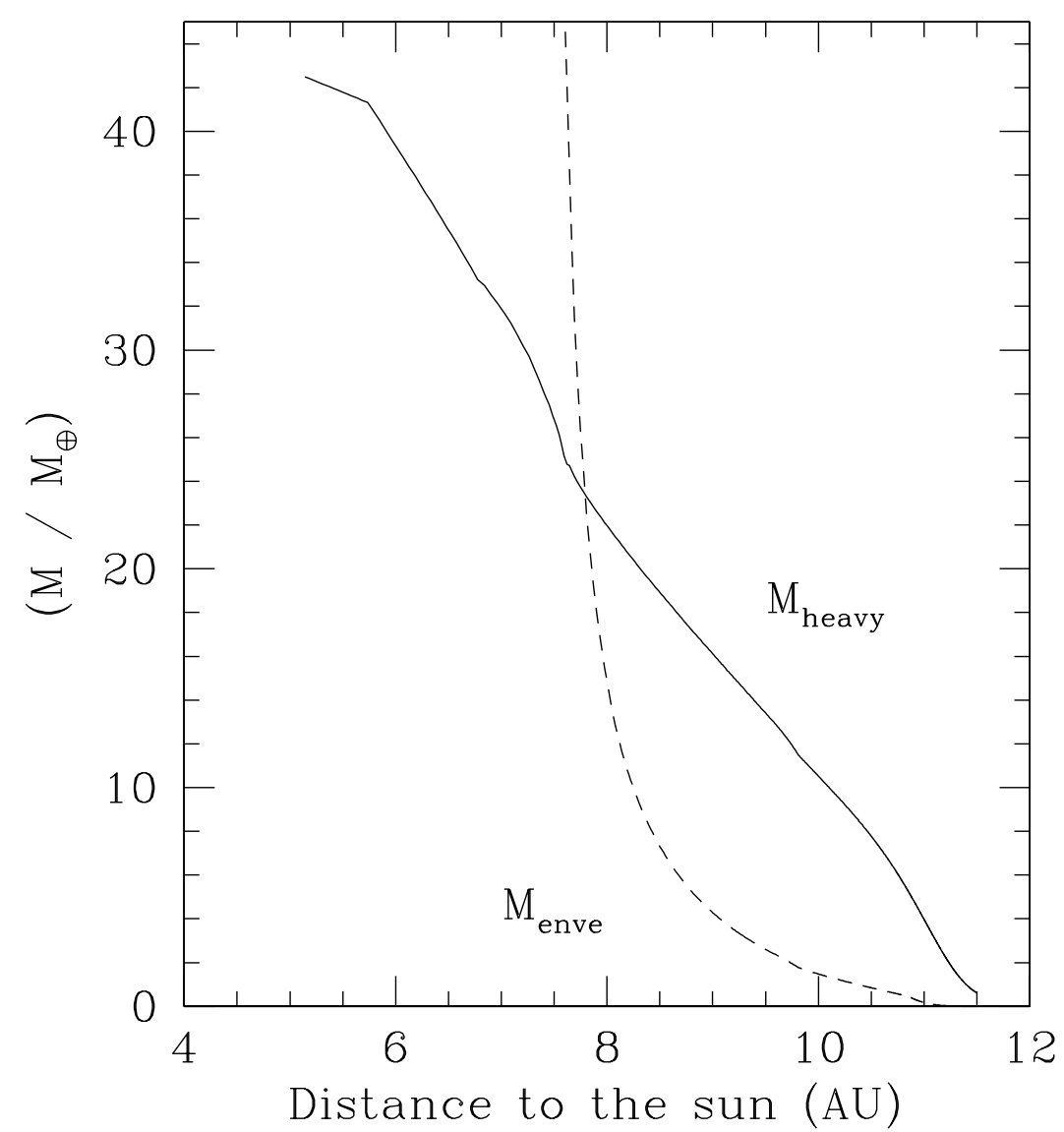

Figure 6. Mass of accreted material onto proto-Jupiter as a function of the distance to the Sun. $M_{\text {heavy }}$ and $M_{\text {enve }}$ represent respectively the mass of accreted planetesimals and the mass of accreted gas.

\subsubsection{Composition of Planetesimals Accreted During the Formation of Proto-Jupiter}

Current scenarios of formation of the solar nebula consider that ices and gases present in the presolar cloud fell onto the disk during the collapse of the cloud, and that most of ices vaporized in the nebula within $30 \mathrm{AU}$ (Chick and Cassen, 1997). This hypothesis is in agreement with the work of Mousis et al. (2002) who have shown that $\mathrm{CO} / \mathrm{CH}_{4}$ and $\mathrm{N}_{2} / \mathrm{NH}_{3}$ ratios in vapor phase in the solar nebula remain quasi identical to the interstellar values. According to Alibert et al. (2004b), we assume that $\mathrm{C}$ is present in vapor phase in the subnebula, in three species, namely $\mathrm{CO}_{2}, \mathrm{CO}$ and $\mathrm{CH}_{4}$. The adopted abundances ratio is here equal to 20/10/1. Regarding nitrogen, we assume that it is present in the form of $\mathrm{N}_{2}$ and $\mathrm{NH}_{3}$, the ratio $\mathrm{N}_{2} / \mathrm{NH}_{3}$ being a free parameter (see Alibert et al., 2004b, for a discussion).

We assume that the amount of available water along the migration pathway of proto-Jupiter is at least high enough to allow the trapping of the volatile species 
TABLE I

Calculations of the ratios of trapped masses of volatiles to the mass of $\mathrm{H}_{2} \mathrm{O}$ ice in planetesimals formed at 5 and $20 \mathrm{AU}$ in the solar nebula. Gas phase abundance of $\mathrm{H}_{2} \mathrm{O}$ is given in text and gas phase abundance of volatiles are assumed to be solar (Anders and Grevesse, 1989) with $\mathrm{CO}_{2} / \mathrm{CO} / \mathrm{CH}_{4}=20 / 10 / 1$ and with $\mathrm{N}_{2} / \mathrm{NH}_{3}=1$ or 10 in vapor phase in the solar nebula.

\begin{tabular}{lcc}
\hline & \multicolumn{1}{c}{$5 \mathrm{AU}$} & $20 \mathrm{AU}$ \\
& $\mathrm{N}_{2} / \mathrm{NH}_{3}=1 / \mathrm{N}_{2} / \mathrm{NH}_{3}=10$ & $\mathrm{~N}_{2} / \mathrm{NH}_{3}=1 / \mathrm{N}_{2} / \mathrm{NH}_{3}=10$ \\
\hline $\mathrm{CO}_{2} / \mathrm{H}_{2} \mathrm{O}$ & $7.12 \times 10^{-1} / 6.90 \times 10^{-1}$ & $6.90 \times 10^{-1} / 6.67 \times 10^{-1}$ \\
$\mathrm{CO} / \mathrm{H}_{2} \mathrm{O}$ & $1.53 \times 10^{-1} / 1.48 \times 10^{-1}$ & $1.54 \times 10^{-1} / 1.49 \times 10^{-1}$ \\
$\mathrm{CH}_{4} / \mathrm{H}_{2} \mathrm{O}$ & $1.02 \times 10^{-2} / 9.91 \times 10^{-3}$ & $9.97 \times 10^{-3} / 9.64 \times 10^{-3}$ \\
$\mathrm{~N}_{2} / \mathrm{H}_{2} \mathrm{O}$ & $4.78 \times 10^{-2} / 6.66 \times 10^{-2}$ & $4.83 \times 10^{-2} / 6.67 \times 10^{-2}$ \\
$\mathrm{NH}_{3} / \mathrm{H}_{2} \mathrm{O}$ & $4.78 \times 10^{-2} / 6.35 \times 10^{-3}$ & $4.50 \times 10^{-2} / 6.22 \times 10^{-3}$ \\
$\mathrm{H}_{2} \mathrm{~S} / \mathrm{H}_{2} \mathrm{O}$ & $4.30 \times 10^{-2} / 4.17 \times 10^{-2}$ & $4.23 \times 10^{-2} / 4.09 \times 10^{-2}$ \\
$\mathrm{Ar} / \mathrm{H}_{2} \mathrm{O}$ & $4.29 \times 10^{-3} / 4.16 \times 10^{-3}$ & $4.45 \times 10^{-3} / 4.30 \times 10^{-3}$ \\
$\mathrm{Kr} / \mathrm{H}_{2} \mathrm{O}$ & $2.29 \times 10^{-6} / 2.22 \times 10^{-6}$ & $2.33 \times 10^{-6} / 2.26 \times 10^{-6}$ \\
$\mathrm{Xe} / \mathrm{H}_{2} \mathrm{O}$ & $3.13 \times 10^{-7} / 3.03 \times 10^{-7}$ & $3.12 \times 10^{-7} / 3.02 \times 10^{-7}$ \\
\hline
\end{tabular}

considered under the form of hydrates or clathrate hydrates. Assuming $\mathrm{N}_{2} / \mathrm{NH}_{3}=$ 1 in the gas phase of the solar nebula and solar abundances (Anders and Grevesse, 1989), the minimum required abundance of water at 5 and $20 \mathrm{AU}$ is 1.85 and 1.95 times the solar water abundance $\left(\mathrm{H}_{2} \mathrm{O} / \mathrm{H}_{2} \sim 5.54 \times 10^{-4}\right.$ in the nebula, with $\mathrm{CO}_{2} / \mathrm{CO} / \mathrm{CH}_{4}=20 / 10 / 1$ in the gas phase), respectively. If $\mathrm{N}_{2} / \mathrm{NH}_{3}=10$ in vapor phase in the solar nebula, the corresponding values are 1.91 and 2.02 at 5 and 20 $\mathrm{AU}$, respectively.

Table I gives the values of the different volatiles to $\mathrm{H}_{2} \mathrm{O}$ mass ratios in planetesimals for $\mathrm{N}_{2} / \mathrm{NH}_{3}=1$ and 10 in the gas phase and for the corresponding minimum abundances of water at 5 and $20 \mathrm{AU}$ in the solar nebula. Since these ratios remain practically identical whatever the distance to the Sun between 5 and 20 AU for given abundances of species, one can assume that icy planetesimals that took part in the formation of proto-Jupiter during its migration shared the same composition in volatiles than those formed at $5 \mathrm{AU}$. The results presented in this section will then not depend on the starting point of the embryo that will form Jupiter, provided it starts its formation process below $20 \mathrm{AU}$.

\subsubsection{The Enrichment in Volatiles in Jupiter's Envelope}

Knowing the amount of planetesimals accreted, as well as their composition, we can calculate the abundance of $\mathrm{C}, \mathrm{N}, \mathrm{Ar}, \mathrm{Xe}$ and $\mathrm{S}$ in the atmosphere of our final planet. 
TABLE II

Comparison of the observed enrichments in volatiles in Jupiter with values calculated from different masses of ices (water and volatiles) vaporized in the envelope of proto-Jupiter ( $\left.M_{\text {ices }}\right)$.

\begin{tabular}{lccc}
\hline Species & Observed & $M_{\text {ices }} / M_{\oplus}=32^{c}$ & $M_{\text {ices }} / M_{\oplus}=27.6^{d}$ \\
\hline $\mathrm{Ar}$ & $2.5 \pm 0.5^{a}$ & 2.32 & 2.00 \\
$\mathrm{Kr}$ & $2.7 \pm 0.5^{b}$ & 2.55 & 2.20 \\
$\mathrm{Xe}$ & $2.5 \pm 0.7^{b}$ & 3.31 & 2.86 \\
$\mathrm{C}$ & $2.9 \pm 0.5^{b}$ & 3.68 & 3.18 \\
$\mathrm{~N}$ & $3.6 \pm 0.8^{b}$ & 2.80 & 2.84 \\
$\mathrm{~S}$ & $2.5 \pm 0.15^{b}$ & 2.65 & 2.65 \\
\hline
\end{tabular}

${ }^{a}$ Mahaffy et al. (2000), ${ }^{b}$ Atreya et al. (1999)

${ }^{c}$ mass of ices required to fit the observed enrichments with $\mathrm{N}_{2} / \mathrm{NH}_{3}=10$ in vapor phase in the solar nebula

${ }^{d}$ mass of ices required to fit the observed enrichments with $\mathrm{N}_{2} / \mathrm{NH}_{3}=1$

in vapor phase in the solar nebula

TABLE III

Calculated masses of water, volatiles (excluding water), total ices, rocks and total mass of heavy elements $\left(\mathrm{M}_{\mathrm{Z}}\right)$ trapped in the envelope of Jupiter and constraint from Guillot et al. (2004). Masses are calculated for $I / R=4$ and different values of $\mathrm{N}_{2} / \mathrm{NH}_{3}$ in vapor phase in the solar nebula.

\begin{tabular}{lccc} 
& \multicolumn{2}{c}{$\mathrm{I} / \mathrm{R}=4$} & Guillot et al. (2004) \\
$\mathrm{N}_{2} / \mathrm{NH}_{3}$ & 10 & 1 & \\
\hline $\mathrm{M}_{\text {water }} / M_{\oplus}$ & 16 & 13.5 & \\
$\mathrm{M}_{\text {volatiles }} / M_{\oplus}$ & 16 & 14.1 & \\
$\mathrm{M}_{\text {ices }} / M_{\oplus}$ & 32 & 27.6 & $\leq 42$ \\
$\mathrm{M}_{\text {rocks }} / M_{\oplus}$ & 8 & 6.9 & \\
$\mathrm{M}_{\mathrm{Z}} / M_{\oplus}$ & 40 & 34.5 & \\
\hline
\end{tabular}

We first adopt $\mathrm{N}_{2} / \mathrm{NH}_{3}=10$ in vapor phase. Using the results of our model, we have tried to reproduce the observed abundances of volatiles (see Table II). While the total mass of heavy elements required in the envelope of Jupiter $\left(40 M_{\oplus}\right.$ assuming a planetesimal ices/rocks (I/R) ratio equal to 4 , see Table III) is almost compatible with our model and is below the upper limit defined by Guillot $e t$ al. (2004), we cannot fit the measured enrichment in carbon. Moreover, we obtain a 
slightly higher value than the upper limit for Xe and an excess of abundance for $\mathrm{S}$. This excess of abundance for $\mathrm{S}$ results from the fact that this element was assumed to be exclusively in the form of $\mathrm{H}_{2} \mathrm{~S}$ in the solar nebula. Since it has been shown that some $\mathrm{H}_{2} \mathrm{~S}$ may have been consumed in the corrosion of $\mathrm{Fe}$ alloy grains at high temperature ranges in the solar nebula to form troilite FeS (Fegley, 2000), one can argue that the abundance of $\mathrm{H}_{2} \mathrm{~S}$ was subsolar in vapor phase in the solar nebula prior to its incorporation in icy planetesimals. Under this assumption, the abundance of $\mathrm{H}_{2} \mathrm{~S}$ has been revised down to fit the measured upper value (0.62 times the solar abundance for $\mathrm{N}_{2} / \mathrm{NH}_{3}=10$ in vapor phase).

Adopting now $\mathrm{N}_{2} / \mathrm{NH}_{3}=1$ in vapor phase in the solar nebula, the minimum mass of ices required to fit the enrichments in volatiles is $27.6 M_{\oplus}$, including $13.5 M_{\oplus}$ of water and $14.1 M_{\oplus}$ of volatiles. This corresponds to $34.5 M_{\oplus}$ of heavy elements in the envelope of Jupiter with $\mathrm{I} / \mathrm{R}=4$. This value is compatible with the ones derived from Guillot et al. (2004) but is slightly lower than what we obtained in our simulation $\left(\sim 36 M_{\oplus}\right)$. However, given the uncertainties on the final value of heavy elements, the difference between the two values is probably not significant.

Finally, adopting, as in Hersant et al. (2004), $\mathrm{CO}_{2} / \mathrm{CO} / \mathrm{CH}_{4}=0 / 10 / 1$ and $\mathrm{N}_{2} / \mathrm{NH}_{3}$ $=10$ in the gas phase of the solar nebula, we can also fit the observed enrichments. However, in that case, at least $37.6 M_{\oplus}$ of water and $10 M_{\oplus}$ of volatiles are required in the envelope of Jupiter. Hence, whatever the adopted I/R ratio, the value of heavy elements required $\left(>47.6 M_{\oplus}\right)$ always exceeds the highest mass of heavy elements (42 $M_{\oplus}$ ) allowed by the internal structure models of Jupiter by Guillot et al. (2004).

\section{Summary and Discussion}

Our calculations show that the formation of giant planets, can be sped-up if one takes into account the effect of migration. This is mainly due to the suppression of phase 2 described in Pollack et al. (1996). We then obtain formation timescales that are compatible with the observed lifetime of protoplanetary disks, without having to consider disks significantly more massive than the minimum mass solar nebula. This effect of course, does not preclude other effects such as reduction of opacity (Hubickyj et al., 2003), formation of vortices prior to planet formation (Klahr and Bodenheimer, 2003) or fragmentation of planetesimals (Inaba et al., 2003) to further reduce this timescale.

Using this model, we are able to model the formation of a Jupiter-like planet, with final properties in good agreement with the ones of Jupiter (core mass, mass of heavy elements, mass of gas, and distance to the sun). We note however that the final mass of heavy elements accreted is a function of the ratio of ejection to accretion rate. A change in this ratio woul modify the accretion rate of solids towards the end of the formation process but not during the early phases. More work is needed to quantify this effect. 
The model presented here starts with an embryo located at some arbitrary distance from the star. The precise location depends on the actual type I migration rate, and may be between $\sim 10$ to $\sim 15 \mathrm{AU}$ for the rates considered here. However one can wonder how likely it is to find embryos at such large distances (Thommes et al., 2003). On the other hand, simulations by Weidenschilling et al. (2004) show that an embryo can be ejected from inner regions in a relatively short timescale (of the order or less than $1 \mathrm{Myr}$ ).

In our simulation forming Jupiter, at the time the planet reaches the current orbit of Saturn, its mass is $\sim 30 M_{\oplus}$ so that perturbations on other objects (such as the Kuiper Belt Objects) will remain small. A planet formed from an embryo initially located much further away (with an increased type I migration rate) might perturb the exterior part of the solar system, and not be consistent with the existence of a cold Kuiper Belt. More calculations are needed to adress this point, which might set an upper limit on the total migration of the planet. Similarly, the effect of migrating giant planets on the terrestrial planet formation, especially in the case of giant planets crossing this region, remains an important issue.

In the framework of our current model, we can reproduce the results on Jupiter derived by Guillot et al. (2004), regarding the mass of the core and the total mass of heavy elements accreted. Moreover, assuming ices/rocks ratio of accreted planetesimals equal to 4 , and that $\mathrm{CO}_{2}$ was present in vapor phase in the outer solar nebula, with $\mathrm{CO}_{2} / \mathrm{CO} \sim 2$, a value consistent with ISM measurements (Gibb et al., 2004), we can explain the enrichments in volatiles observed by the Galileo probe, while being compatible with Guillot et al. (2004).

Finally, it appears very difficult to form a planet, and to prevent it from spiraling into the sun if the amount of type I migration as computed today is not reduced by a factor of at least 10 . Our results then strongly suggest that there might still be a serious problem in our understanding of this type of migration.

\section{Acknowledgements}

This work was supported in part by the Swiss National Science Foundation. O.M. was supported by an ESA external fellowship, and this support is gratefully acknowledged.

\section{References}

Alibert, Y., Mordasini, C., and Benz, W.: 2004a, 'Migration and giant planet formation', Astron. Astrophys. 417, L25-L28.

Alibert, Y., Mousis, O., and Benz, W.: 2004b, 'Volatiles enrichments in Jupiter', in preparation.

Allamandola, L.J., Bernstein, M.P., Sandford, S.A., and Walker, R.L.: 1999, 'Evolution of interstellar ices', Space Sci. Rev. 90, 219-232.

Anders, E., and Grevesse, N.: 1989, 'Abundances of the elements - meteoritic and solar', Geochim. Cosmochim. Acta 53, 197-214. 
Atreya, S.K., Wong, M.H., Owen, T.C., Mahaffy, P.R., Niemann, H.B., de Pater, I., Drossart, P., and Encrenaz, T.: 1999, 'A comparison of the atmospheres of Jupiter and Saturn: Deep atmospheric composition, cloud structure, vertical mixing, and origin', Planet. Space Sci. 47 1243-1262.

Bell, K.R. and Lin, D.N.C.: 1994, 'Using FU Orionis outbursts to constrain self-regulated protostellar disk models', Astrophys. J. 427, 987-1004.

Bodenheimer, P. and Pollack, J.B.: 1986, 'Calculations of the accretion and evolution of giant planets: The effects of solid cores', Icarus 67, 391-408.

Boss, A.P.: 2002, 'Evolution of the solar nebula: V. Disk instabilities with varied thermodynamics', Astrophys. J. 576, 462-472.

Boss, A.P.: 2004, 'Rapid formation of outer giant planets by disk instability', Astrophys. J. 599, $577-581$.

Chabrier, G., Saumon, D., Hubbard, W.B., and Lunine, J.I.: 1992, 'The molecular-metallic transition of hydrogen and the structure of Jupiter and Saturn', Astrophys. J. 391, 817-826.

Chick, K.M. and Cassen, P.: 1997, 'Thermal processing of interstellar dust grains in the primitive solar environment', Astrophys. J. 447, 398-409.

Fegley, B., Jr.: 2000, 'Kinetics of gas-grain reaction in the solar nebula', Space Sci. Rev. 92, 177-200.

Gautier, D., Hersant, F., Mousis, O., and Lunine J.I.: 2001a, 'Enrichments in volatiles in Jupiter: A new interpretation of the Galileo measurements', Astrophys. J. 550, L227-L230.

Gautier, D., Hersant, F., Mousis, O., and Lunine J.I.: 2001b, 'Erratum: Enrichments in volatiles in Jupiter: A new interpretation of the Galileo measurements', Astrophys. J. 559, L183-L183.

Gibb, E.L., Whittet, D.C.B., Boogert, A.C.A., and Tielens, A.G.G.M.: 2004, 'Interstellar ice: The infrared space observatory legacy', Astrophys. J. Supp. 151, 35-73.

Greenzweig, Y. and Lissauer, J.J.: 1992, 'Accretion rates of protoplanets. II - Gaussian distributions of planetesimal velocities', Icarus 100, 440-463.

Guillot, T. and Gladman, B.: 2000, 'Delivery of planetesimals and Giant planet formation', in F. Garzón, C. Eiroa, D. de Winter, and T.J. Mahoney (eds.), Disks, Planetesimals, and Planets, ASP Conf. Proc. 219, 475.

Guillot, T., Stevenson, D.J., Hubbard, W.B., and Saumon, D.: 2004, 'The interior of Jupiter', in F. Bagenal, T.E. Dowling, and W.B. McKinnon (eds.), Jupiter, in press.

Haisch, K.E., Lada, E.A., and Lada, C.J.: 2001, 'Disk frequencies and lifetimes in young clusters', Astrophys. J. 553, L153-L156.

Henderson, C.B.: 1976, 'Drag coefficient of spheres in continuum and rarefied flows', AIAA Journal 14, 707-707.

Hersant, F., Gautier, D., and Huré, J.M.: 2001, 'A 2-D model for the primordial nebula constrained by $\mathrm{D} / \mathrm{H}$ measurements in the solar system: implications for the formation of giant planets', Astrophys. J. 554, 391-407.

Hersant, F., Gautier, D., and Lunine J.I.: 2004, 'Enrichment in volatiles in the giant planets of the solar system', Planet. Space Sci. 52, 623-641.

Hills, J.G. and Goda, M.P.: 1993, 'The fragmentation of small asteroids in the atmosphere', Astron. J. 105, 1114-1144.

Horner, J., Alibert, Y., and Benz, W.: 2004, in preparation.

Hubickyj, O., Bodenheimer, P., and Lissauer, J.: 2003, 'Small Core for Jupiter using the Core Instability Model', Proc.35th DPS meeting, 25.06.

Inaba, S., Wetherill, G.W., and Ikoma, M.: 2003, 'Formation of gas Giant planets: Core accretion models with fragmentation and planetary envelope', Icarus 166, 46-62.

Ida, S. and Lin, D.N.C.: 2004, 'Toward a deterministic model of planetary formation. I. A desert in the mass and semimajor axis distributions of extrasolar planets', Astrophys. J. 604, 388-413.

Klahr, H. and Bodenheimer, P.: 2003, 'A three phase model for planet formation - the formation of a planet in the eye of a hurricane', in M. Fridlund and T. Henning (eds.), Earths: DARWIN/TPF and the Search for Extrasolar Terrestrial Planets, ESA SP-539, 481-483. 
Lin, D.N.C. and Papaloizou, J.: 1985, 'On the dynamical origin of the solar system', in D.C. Black and M.S. Matthews (eds.), Protostars and planets II, Tucson, AZ, University of Arizona Press, 981-1072.

Lin, D.N.C. and Papaloizou, J.: 1986, 'On the tidal interaction between protoplanets and the protoplanetary disk. III - Orbital migration of protoplanets', Astrophys. J. 309 846-857.

Lin, D.N.C., Bodenheimer, P., and Richardson, D.C.: 1996, 'Orbital migration of the planetary companion of 51 Pegasi to its present location', Nature 380 606-607.

Lunine, J.I. and Stevenson, D.J.: 1985, 'Thermodynamics of clathrate hydrate at low and high pressures with application to the outer solar system', Astrophys. J. Supp. 58, 493-531.

Mahaffy, P.R., Niemann, H.B., Alpert, A., Atreya, S.K., Demick, J., Donahue, T.M., Harpold, D.N., and Owen, T.C.: 2000, 'Noble gas abundance and isotope ratios in the atmosphere of Jupiter from the Galileo Probe Mass Spectrometer', J. Geophys. Res. 105, 15,061-15,072.

MacLow, M.M. and Zahnle, K.J.: 1994, 'Explosion of comet Shoemaker-Levy 9 on entry into the Jovian atmosphere', Astrophys. J. 434, L33-L36.

Menou, K. and Goodman, J.: 2003, 'Low-mass proto-planet migration in T-Tauri $\alpha$-disks', Astrophys. J. 606, 520-531.

Mousis, O., Gautier, D., and Bockelée-Morvan, D.: 2002, 'An evolutionary turbulent model of Saturn's subnebula: Implications for the origin of the atmosphere of Titan', Icarus 156, 162-175.

Mousis, O. and Gautier, D.: 2004, 'Constraints on the presence of volatiles in Ganymede and Callisto from an evolutionary turbulent model of the Jovian subnebula', Planet. Space Sci. 52, 361-370.

Nelson, R.P. and Papaloizou, J.C.B.: 2003, 'The interaction of planets with a disc with MHD turbulence IV: Migration rates of embedded protoplanets', Mon. Not. Roy. Astron. Soc. 350, 849-864.

Owen, T.C., Mahaffy, P.R., Niemann, H.B., Atreya, S.K., Donahue, T.M., Bar-Num, A., and de Pater, I.: 1999, 'A low temperature origin for the planetesimals that formed Jupiter', Nature 402, 269270.

Papaloizou, J.C.B. and Terquem, C.: 1999, 'Critical protoplanetary core masses in protoplanetary disks and the formation of short-period Giant planets', Astrophys. J. 521, 823-838.

Pollack, J.B., Hubickyj, O., Bodenheimer, P., Lissauer, J.J., Podolak, M., and Greenzweig, Y.: 1996, 'Formation of the giant planets by concurrent accretion of solids and gas', Icarus 124, 62-85.

Tanaka, H., Takeuchi, T. and Ward, W.R.: 2002, 'Three-dimensional interaction between a planet and an isothermal gaseous disk. I. Corotation and Lindblad torques and planet migration', Astrophys. J. 565, 1257-1274.

Thommes, E.W., Duncan, M.J., and Levison, H.F.: 2003, 'Oligarchic growth of giant planets', Icarus 161, 431-455.

Trilling, D.E., Benz, W., Guillot, T., Lunine, J.I., Hubbard, W.B., and Burrows, A.: 1998, 'Orbital evolution and migration of Giant planets: Modeling extrasolar planets', Astrophys. J. 500, 428 439.

Veras, D. and Armitage, P.J.: 2003, 'Outward migration of extrasolar planets to large orbital radii', Mon. Not. Roy. Astron. Soc. 347, 613-624.

Ward, W.R.: 1997, 'Survival of planetary systems', Astrophys. J. 482 L211-L217.

Weidenschilling, S.J., Marzari, F., and Davis, D.R.: 2004, 'Accretion of the Outer planets: Oligarchy or monarchy?', Lunar Planet. Inst. Conf. Abstr. 35, 1174.

Zahnle, K.J.: 1992, 'Airburst origin of dark shadows on Venus', J. Geophys. Res. 97, 10243-10255.

Address for Offprints: Yann Alibert, Physikalisches Institut, Hallerstrasse 6, CH-3012 Bern, Switzerland; yann.alibert@phim.unibe.ch 